\title{
Laminectomy versus Unilateral Hemilaminectomy for the Removal of Intraspinal Schwannoma: Experience of a Single Institution and Review of Literature
}

\author{
Mauro Dobran ${ }^{1}$ Riccardo Paracino ${ }^{1}$ Davide Nasi ${ }^{2}$ Denis Aiudi ${ }^{1}$ Mara Capece ${ }^{1}$ Erika Carrassi ${ }^{1}$ \\ Simona Lattanzi ${ }^{3} \quad$ Alessandro DI Rienzo ${ }^{1} \quad$ Maurizio lacoangeli ${ }^{1}$ \\ ${ }^{1}$ Department of Neurosurgery, Università Politecnica delle Marche, \\ Ancona, Italy \\ ${ }^{2}$ Department of Neurosurgery, Azienda Ospedaliero-Universitaria di \\ Address for correspondence Mauro Dobran, Department of \\ Neurosurgery, Università Politecnica delle Marche, via conca 71 \\ Modena Ospedale Civile di Baggiovara, Modena, Italy \\ ${ }^{3}$ Department of Neurology, Università Politecnica delle Marche, \\ Ancona, Italy \\ J Neurol Surg A Cent Eur Neurosurg 2021;82:552-555.
}

\begin{abstract}
Background and Study Aims Spinal schwannomas are benign slow-growing tumors, and gross total resection is the gold standard of treatment. The conventional surgical approach is laminectomy, which provides a wide working area. Today minimally invasive surgery (MIS) is popular because it is associated with shorter hospital stay, less operative blood loss, minimized tissue traumas and relative postoperative pain, and, and spine surgery, avoidance of spinal instability.

Material and Methods From January 2016 to December 2019, we operated on 40 patients with spinal intradural extramedullary tumor (schwannoma) with laminectomy or hemilaminectomy. Baseline medical data, including patients' sex and age, tumor location, days of postoperative bed rest, operative time, length of hospitalization, and 1-month visual analog scale (VAS) value were collected and analyzed. Data analysis was performed using STATA/IC 13.1 statistical package (StataCorp LP, College Station, Texas, United States).

\section{Keywords}

- hemilaminectomy

- Spinal schwannomas

- spinal intradural extramedullary tumors

- spinal minimally invasive approaches

- laminectomy

Results Hemilaminectomy was associated with faster operative time $(p<0.001)$, shorter postoperative time spent in bed $(p<0.001)$, and shorter hospitalization $(p<0.001)$. At 1 -month follow-up, the mean VAS score was $4.6(1.7)$ among the laminectomy patients and $2.5(1.3)$ among the hemilaminectomy patients $(p<0.001)$. Postoperative complications occurred in 1 (7.7\%) and 7 (25.9\%) patients in the hemilaminectomy and laminectomy groups, respectively $(p=0.177)$.

Conclusions Unilateral hemilaminectomy has significant advantages compared with laminectomy in spinal schwannoma surgery including shorter operative time, less time spent in bed, shorter hospitalization, and less postoperative pain.
\end{abstract}

\section{Introduction}

Spinal schwannomas are benign slow-growing tumors, and gross total resection is the gold standard of treatment. The conventional surgical approach is laminectomy, which pro-

received

April 8, 2020

accepted after revision

September 22, 2020

published online

April 12, 2021 vides a wide working area. However, minimally invasive approaches, such as hemilaminectomy, minimize tissue traumas and avoid spinal instability, but they may be relatively contraindicated in large spinal tumors. ${ }^{1,2}$ Because of (c) 2021. Thieme. All rights reserved.

Georg Thieme Verlag KG,

Rüdigerstraße 14,

70469 Stuttgart, Germany
DOI https://doi.org/ 10.1055/s-0041-1722968. ISSN 2193-6315. 
the weak adherence of a spinal schwannoma tumor to surrounding tissues, excision via a hemilaminectomy appears feasible. In this article, we describe and discuss our experience and results about a series of patients with spinal schwannoma and operated on with laminectomy or hemilaminectomy.

\section{Material and Methods}

From January 2016 to December 2019, we operated 40 patients with a spinal intradural schwannoma via laminectomy or hemilaminectomy. In all cases, surgery was indicated for the treatment of the symptoms (neurologic deficit, intractable pain, numbness) and the diagnosis was obtained by contrastenhanced magnetic resonance imaging (MRI). In the last 18 months of the study, spinal schwannoma was treated exclusively via hemilaminectomy ${ }^{3}$; only very large schwannomas were treated via laminectomy.

Baseline medical data, including patients' sex, age, tumor location and volume, overall operative time, length of postoperative immobilization, duration of hospitalization, 1-month post-op visual analog scale (VAS) value comorbidities, and preoperative American Society of Anesthesiologists (ASA) physical status classification system were collected and analyzed ( - Table 1). Tumor size was measured using the widest diameter in three planes and the volume was calculated (ellipsoid method volume $=\mathrm{D} 1 \times \mathrm{D} 2 \times \mathrm{D} 3 / 2$ ). The diagnosis of schwannoma was confirmed in all cases at histopathology. All procedures were performed by two expert neurosurgeons.

\section{Operative Technique}

The patient was placed in the prone position under general anesthesia. A midline skin incision was centered on the radiograph marker positioned in the spinous process over the tumor. Intraoperative neurophysiologic monitoring was not used, in 5

Table 1 Baseline characteristics of patients

\begin{tabular}{|c|c|c|c|}
\hline & $\begin{array}{l}\text { Laminectomy } \\
(n=27)\end{array}$ & $\begin{array}{l}\text { hemilaminectomy } \\
(n=13)\end{array}$ & $p$ value \\
\hline Age $(y)$ & $66.2(13.4)$ & $60.0(9.7)$ & $0.144^{\mathrm{a}}$ \\
\hline Male sex & 7 (25.9) & $1(7.7)$ & $0.177^{b}$ \\
\hline \multicolumn{4}{|c|}{ Site of surgery } \\
\hline Cervical & $3(11.1)$ & $5(38.5)$ & \multirow[t]{3}{*}{$0.105^{b}$} \\
\hline Thoracic & $18(66.7)$ & $5(38.5)$ & \\
\hline Lumbar & $6(22.2)$ & $3(23.0)$ & \\
\hline $\begin{array}{l}\text { Tumor size } \\
(\mathrm{ccm})\end{array}$ & $\begin{array}{l}0.61 \\
{[0.35-0.85]}\end{array}$ & $0.60[0.45-0.80]$ & $0.675^{c}$ \\
\hline $\begin{array}{l}\text { Baseline } \\
\text { ASA status }\end{array}$ & $2.1(0.7)$ & $2.2(0.6)$ & $0.980^{\mathrm{a}}$ \\
\hline
\end{tabular}

Abbreviation: ASA, American Society of Anesthesiologists physical status classification system.

Note: Data are mean (SD) for continuous variables, and $n(\%)$ for categorical variables.

a Two-sample $t$-test.

${ }^{\mathrm{b}}$ Chi-squared test.

'Mann-Whitney test.

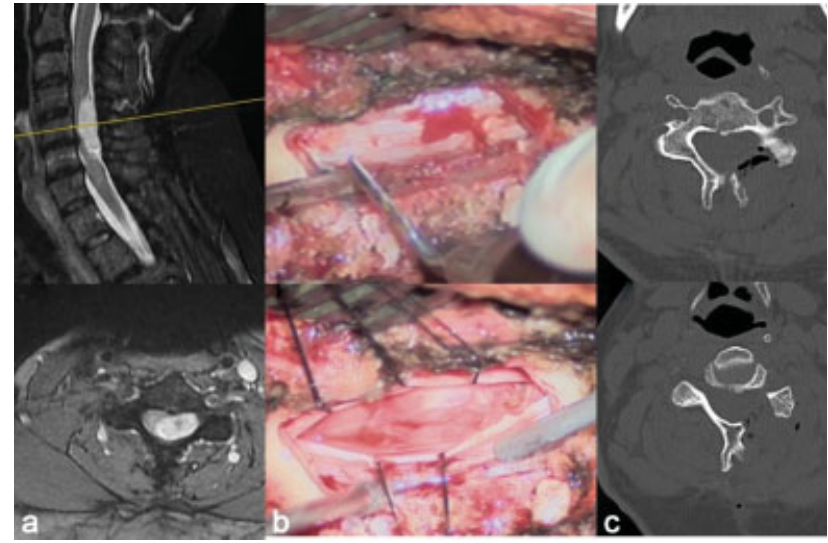

Fig. 1 (a) Preoperative cervical magnetic resonance imaging (MRI) study. (b) Intraoperative unilateral hemilaminectomy with tumor exposure. (c) Postoperative computed tomography (CT) scan study.

out of 40 cases. In hemilaminectomy, the paravertebral muscles of the tumor side were retained to reach the hemilaminae. By means of a high-speed pneumatic drill or piezoelectric device, the hemilaminae were removed to expose the dural sac. In some patients with more lateral tumor, the exposure was completed with a partial facetectomy. The dural sac was opened under operating microscope magnification and the tumor was decompressed internally by the cavitron ultrasonic aspirator. Finally, the capsula was dissected from the surrounding tissues in the majority of cases; when this was not possible, for dorsal schwannomas, the sensitive nerve root was coagulated and cut, whereas for cervical and lumbar tumors, a small tumor residual near the nerve was sometimes left (- Fig. 1). The dura mater was sutured in a watertight fashion with 4-0 silk wire. Fibrin glue and fat were applied epidurally to reinforce the closure. In case of laminectomy, the muscle dissection was bilateral, the laminae were removed, and the dural sac was exposed and opened for tumor removal under the operating microscope (-Fig. 2). Instrumentation for spinal instability was never required.

\section{Statistical Analysis}

Values are presented as mean \pm standard deviation (SD) or median (interquartile range [IQR]) for continuous variables

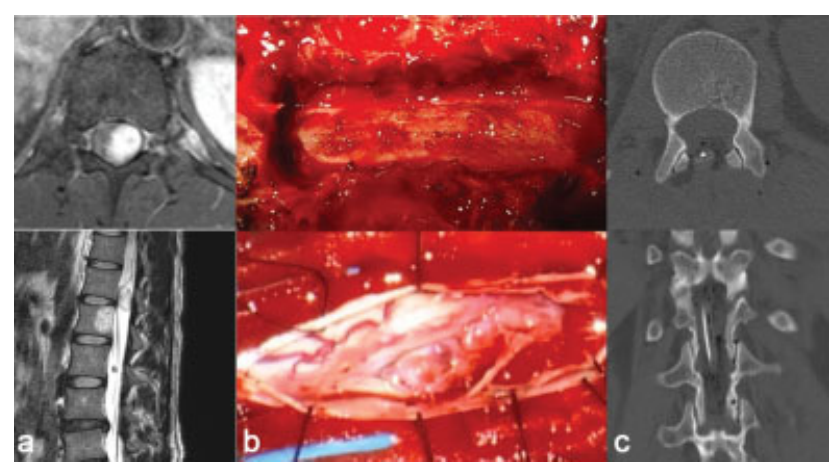

Fig. 2 (a) Preoperative dorsal magnetic resonance imaging (MRI) study. (b) Intraoperative laminectomy with tumor exposure. (c) Postoperative computed tomography (CT) scan study. 
Table 2 Operative outcomes according to surgical intervention type

\begin{tabular}{|l|l|l|l|}
\hline & $\begin{array}{l}\text { Laminectomy } \\
(\boldsymbol{n}=\mathbf{2 7})\end{array}$ & $\begin{array}{l}\text { Hemilaminectomy } \\
(\boldsymbol{n}=\mathbf{1 3})\end{array}$ & $p$ value \\
\hline $\begin{array}{l}\text { Operative } \\
\text { time (min) }\end{array}$ & $\begin{array}{l}160 \\
{[130-240]}\end{array}$ & $90[80-100]$ & $<0.001^{\mathrm{a}}$ \\
\hline $\begin{array}{l}\text { Time spent } \\
\text { in bed (h) }\end{array}$ & $72[70-100]$ & $48[24-70]$ & $<0.001^{\mathrm{a}}$ \\
\hline $\begin{array}{l}\text { Hospitalization } \\
\text { length (d) }\end{array}$ & $12[10-15]$ & $7[5-9]$ & $<0.001^{\mathrm{a}}$ \\
\hline $\begin{array}{l}\text { Follow-up } \\
\text { VAS score }\end{array}$ & $4.6(1.7)$ & $2.5(1.3)$ & $<0.001^{\mathrm{b}}$ \\
\hline
\end{tabular}

Abbreviation: VAS, visual analog scale.

Note: Data are mean (standard deviation [SD]) or median (interquartile range [IQR]) for continuous variables, and $n(\%)$ for categorical variables. ${ }^{a}$ Mann-Whitney test.

${ }^{\mathrm{b}}$ Two-sample $t$-test.

and as the number (percent) of subjects for categorical variables. Univariate comparisons were made through Student's $t$-test, Mann-Whitney test, or chi-squared test, as appropriate (-Table 2). The association between the VAS score at follow-up and the type of surgery (laminectomy vs. hemilaminectomy) was estimated using a linear regression analysis ( - Table 3 ). Age, sex, operative time, tumors size in cubic centimeter $(\mathrm{ccm})$ and level of surgery were included in the multivariate models. Results were considered significant for $p$ values $<0.05$ (two sided). Data analysis was performed using STATA/IC 13.1 statistical package (StataCorp LP, College Station, Texas, United States).

\section{Results}

In total, 40 patients were included, with laminectomy performed in 27 (67.5\%) patients and hemilaminectomy in 13 (32.5\%) patients. Mean age was 64.2 (12.5) years, with a significant preponderance of females ( 32 cases, $80.0 \%$ ). Hemilaminectomy was associated with shorter operative time $(p<0.001)$, faster mobilization $(p<0.001)$, and shorter hospital stay $(<0.001)$. No significant statistical correlation between tumor's volume $(\mathrm{ccm})$ and operative time was found
( $p=0.675 ;-$ Table 1$)$. At follow-up, the mean VAS score was 4.6 (1.7) among patients treated vialaminectomy and 2.5 (1.3) among those treated viahemilaminectomy $(p<0.001)$. At the regression analysis, hemilaminectomy was significantly associated with lower VAS values at follow-up ( $\beta$ coefficient $=-2.02 ; 95 \%$ confidence interval $[\mathrm{CI}]:-3.10$ to -0.93 , $p=0.001 ;{ }_{\text {adj }} \beta=-1.54 ; 95 \% \mathrm{Cl}:-2.95$ to $-0.13, p=0.033$ ), whereas the surgical site was not related to clinical outcome at statistical analysis $(p=0.105)$. Postoperative complications occurred in $1(7.7 \%)$ and 7 (25.9\%) patients in the hemilaminectomy and laminectomy groups, respectively $(p=0.177)$.

\section{Discussion}

Spinal schwannomas are slow-growing tumors originating from myelinated nerve sheaths and classified as intradural extramedullary (IDEM lesions. ${ }^{1}$ Laminectomy extending one level above and one level below the tumor is the commonest surgical approach, ${ }^{2}$ granting adequate exposure, although potentially increasing the risks of postoperative instability, especially at junctional levels. ${ }^{2,3}$ This is particularly true for cervical operations, where kyphosis can cause anterior cord compression and progressive myelopathy. ${ }^{4,5}$

Over the past 15 years, minimally invasive procedures have been gaining popularity due reduction of the impact of the approach on spine stability, with an improvement on postoperative pain and hospital stay. ${ }^{6,7}$ Hemilaminectomy, thus, has become one of the preferred surgical approaches for either spinal degenerative diseases or tumors. ${ }^{8}$

In 1991, Yasargil et al recommended the unilateral approach as the first choice of treatment for removal of intraspinal tumors. Their recommendation was based on the theory that unilateral hemilaminectomy exposes the ventral lateral and dorsal lateral tumors of the spinal canal. ${ }^{9,10}$

Our surgical series of spinal schwannomas compared the unilateral hemilaminectomy approach with conventional laminectomy and it demonstrated the safety and efficacy of the first approach. In our study, 13 of 40 (32.5\%) patients underwent tumor resection via hemilaminectomy. Consistent with reports in other studies, we found that hemilaminectomy was

Table 3 Linear regression model predicting visual analog scale score at follow-up

\begin{tabular}{|l|l|l|l|l|}
\hline \multirow{2}{*}{ Variable } & \multicolumn{2}{|l|}{ Unadjusted } & \multicolumn{2}{|l|}{ Adjusted $^{\text {a }}$} \\
\cline { 2 - 5 } & $\beta$ coefficient $(95 \% \mathrm{Cl})$ & $\boldsymbol{p}$ value & $\beta$ coefficient $(95 \% \mathrm{Cl})$ & $\boldsymbol{p}$ value \\
\hline Age & $0.01(-0.04$ to 0.06$)$ & 0.591 & $0.01(-0.04$ to 0.06$)$ & 0.689 \\
\hline Sex & $0.79(-0.60$ to 2.18$)$ & 0.258 & $0.43(-1.16$ to 2.01$)$ & 0.588 \\
\hline Tumor size & $-0.01(-1.53$ to 1.51$)$ & 0.994 & $0.07(-1.30$ to 1.44$)$ & 0.920 \\
\hline Baseline ASA status & $0.56(-0.33$ to 1.45$)$ & 0.210 & $0.57(-0.50$ to 1.63$)$ & 0.285 \\
\hline Operative time & $0.009(-0.003$ to 0.016$)$ & 0.007 & $0.003(-0.005$ to 0.012$)$ & 0.399 \\
\hline Site of surgery & $0.54(-0.36$ to 1.43$)$ & 0.234 & $0.37(-0.50$ to 1.23$)$ & 0.391 \\
\hline Type of surgery & $-2.02(-3.10$ to -0.93$)$ & 0.001 & $-1.47(-2.91$ to -0.04$)$ & 0.044 \\
\hline
\end{tabular}

Abbreviations: ASA, American Society of Anesthesiologists physical status classification system; $\mathrm{Cl}$, confidence interval.

Note: $\beta$ coefficients with their $95 \% \mathrm{Cls}$ and corresponding $p$ values from linear regression models are shown.

${ }^{a}$ Adjusted for age, sex, operative time, site, and type of surgery. 
associated with faster operative time, shorter postoperative time spent in bed, and shorter hospitalization with statistical significance. ${ }^{1,2,10-12}$ Tumor volumes (ccm) were found to be unrelated to operative time at statistical analysis $(p=0.675)$. Furthermore, at follow-up after 1 month, VAS score in the hemilaminectomy group was lower than that of the laminectomy group; these data were statistically significant. Minimal surgical trauma and preservation of vertebral column integrity and biomechanics are factors, which might have contributed to these findings.

The rationale behind hemilaminectomy for tumor removallies on the preservation of the contralateral hemilamina, muscles, and supraspinous/interspinous ligaments with the integrity of the "tension band." ${ }^{\prime 9}$ In this and other studies, hemilaminectomy was associated with less intraoperative blood loss, less postoperative pain, and preservation of spinal stability with more favorable clinical outcomes. ${ }^{10-12}$ In our experience, a potential disadvantage is the narrow surgical corridor left between the spinous process and the facet joint with risks of dural and nerve root damage or incomplete tumor removal. However, undercutting of the spinous process base, partial facetectomy, and oblique tilting of the operating table allow a safe dissection of the tumor. Yeo et al described another way to get more surgical space by ipsilateral dural tacking and suture with the base of the muscle or fascia near the facet joint. ${ }^{13}$

This study has some limitations, such as the small number of patients, short follow-up, and the retrospective nature. More studies are needed to confirm the findings of this study. However, the minimally invasive approach is relatively simple and straightforward, and provides good results in terms of total resection and postoperative quality of life. Compared with the group treated with laminectomy, patients who underwent hemilaminectomy experienced a shorter hospital stay, less complications, improved wound healing, faster recovery, and less disabling cervical and/or dorsal, lumbar pain. All these benefits allow for earlier mobilization, which is the major target for good postoperative quality of life in patients, particularly elderly patients.

\section{Conclusion}

Unilateral hemilaminectomy has significant advantages in the removal of spinal schwannoma. This approach allows safe and complete resection of schwannomas with good clinical and radiologic results. Hemilaminectomy for intradural tumors offers many benefits such as faster operative time, shorter postoperative time spent in bed, shorter hospitalization, and less postoperative pain.

Conflict of Interest

None declared.

\section{References}

1 Lee SE, Jahng T-A, Kim HJ. Different surgical approaches for spinal schwannoma: a single surgeon's experience with 49 consecutive cases. World Neurosurg 2015;84(06):1894-1902

2 Lu DC, Chou D, Mummaneni PV. A comparison of mini-open and open approaches for resection of thoracolumbar intradural spinal tumors. J Neurosurg Spine 2011;14(06):758-764

3 Gandhi RH, German JW. Minimally invasive approach for the treatment of intradural spinal pathology. Neurosurg Focus 2013; 35(02):E5

4 Asazuma T, Nakamura M, Matsumoto M, Chibo K, Toyama Y. Postoperative changes of spinal curvature and range of motion in adult patients with cervical spinal cord tumors: analysis of 51 cases and review of the literature. J Spinal Disord Tech 2004;17 (03):178-182

5 Ozawa H, Kokubun S, Aizawa T, Hoshikawa T, Kawahara C. Spinal dumbbell tumors: an analysis of a series of 118 cases. J Neurosurg Spine 2007;7(06):587-593

6 Iacoangeli M, Nasi D, Colasanti R, et al. Endoscopic endonasal odontoidectomy with anterior $\mathrm{C} 1$ arch preservation in rheumatoid arthritis: long-term follow-up and further technical improvement by anterior endoscopic C1-C2 screw fixation and fusion. World Neurosurg 2017;107:820-829

7 Oktem IS, Akdemir H, Kurtsoy A, Koç RK, Menkü A, Tucer B. Hemilaminectomy for the removal of the spinal lesions. Spinal Cord 2000;38(02):92-96

8 Iacoangeli M, Gladi M, Di Rienzo A, et al. Minimally invasive surgery for benign intradural extramedullary spinal meningiomas: experience of a single institution in a cohort of elderly patients and review of the literature. Clin Interv Aging 2012;7:557-564

9 Sun CX, Meng XL, Xie SN, Yu Y, Yang HJ, Wu B. Unilateral hemilaminectomy for patients with intradural extramedullary tumors. J Zhejiang Univ Sci B 2011;12(07):575-581

10 Haji FA, Cenic A, Crevier L, Murty N, Reddy K. Minimally invasive approach for the resection of spinal neoplasm. Spine 2011;36 (15):E1018-E1026

11 Tredway TL, Santiago P, Hrubes MR, Song JK, Christie SD, Fessler RG. Minimally invasive resection of intradural-extramedullary spinal neoplasms. Neurosurgery 2006;58(01, Suppl):ONS52-ONS58, discussion ONS52-ONS58

12 Turel MK, D'Souza WP, Rajshekhar V. Hemilaminectomy approach for intradural extramedullary spinal tumors: an analysis of 164 patients. Neurosurg Focus 2015;39(02):E9

13 Yeo DK, Im SB, Park KW, Shin DS, Kim BT, Shin WH. Profiles of spinal cord tumors removed through a unilateral hemilaminectomy. J Korean Neurosurg Soc 2011;50(03):195-200 